gend erforderlich. Deshalb gehört dieses Programm zu Special Olympics. Untersuchungen, Beratung und Information erfolgen in behindertengerechter Weise, wir erreichen aber auch Angehörige und Betreuer. Zusätzlich werden anonymisierte Daten gewonnen, die in eine internationale Datenbank einfließen. Sie stehen uns auch für nationale gesundheitspolitische Diskussionen zur Verfügung.

MMW: Da viele Sportler in Einrichtungen und Werkstätten betreut werden, sollte man eigentlich denken, dass dort intensiv auf die Gesundheit geachtet wird.

Kaschke: Das ist eher ein Problem der gesetzlichen Regelungen. Es steht Menschen mit geistiger Behinderung ja nur das zur Verfügung, was auch anderen von der GKV angeboten wird. Bei der Zahn- und Mundgesundheit enden kostenfreie präventive Leistungen mit dem 18. Lebensjahr, danach fallen diese Patienten aus dem Raster, sie können sich ja meist keine Individualprophylaxe beim Zahnarzt leisten. Und wenn sie bei einem niedergelassenen Arzt erscheinen, bekommt dieser ja keinen Zuschlag, falls statt 15 Minuten eigentlich eine Stunde nötig ist, um herauszufinden, was fehlt, weil Menschen mit geistiger Behinderung ihre Beschwerden oft nicht klar äußern können.

MMW: Für Healthy Athletes brauchen Sie viel medizinisches Fachpersonal. Woher bekommen Sie das?

Kaschke: Alle Gesundheitsprogramme werden verantwortlich durch ehrenamtlich engagierte, speziell fortgebildete Ärzte, Zahnärzte, Physiotherapeuten und Optometristen geleitet. Wir versuchen, vor Ort weitere ehrenamtliche Freiwillige als Helfer zu gewinnen. Sie werden von uns trainiert und angeleitet. Nach dieser Erfahrung sind sie auch eher bereit, Menschen mit geistiger Behinderung in ihrer Praxis zu behandeln und zu beraten. Damit decken wir ein Ausbildungs- und Weiterbildungsdefizit $a b$. Unterstützt werden wir bei der Suche von den jeweiligen Ärztekammern. Das klappt sehr gut, denn beim Sport ist das Thema geistige Behinderung positiv besetzt. Wir schaffen da nicht Mitleid, hier herrscht Freude und eine entspannte Atmosphäre.

- Interview: Thomas Müller

\title{
SCHWEDISCHE KOHORTENSTUDIE Kräftige junge Männer leben länger
}

- Schlaffe Jungs sterben früher, trotz Idealwerten bei BMI und Blutdruck. In einer prospektiven Kohortenstudie untersuchten schwedische Wissenschaftler den Zusammenhang zwischen der jugendlichen Kraft und dem Risiko eines Todes vor dem 55. Geburtstag. Hierzu führten sie an über 1,1 Millionen Jugendlichen zwischen 16 und 19 Jahren Tests zur Muskelstärke durch (u.a. Handgrip-Strength-Test, Knee-ExtensionTest), maßen Blutdruck und bestimmten den Body-Mass-Index (BMI). Anschließend wurden die Probanden über durchschnittlich 24 Jahre hinweg verfolgt.

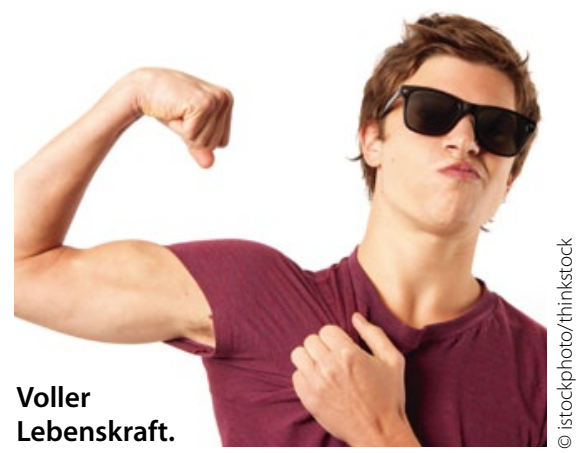

Im Studienverlauf starben 26145 Männer. Haupttodesursache war mit 25,9\% ein Unfall, gefolgt von Suizid (22,3\%), Krebs $(14,9 \%)$ und Herz-Kreislauf-Erkrankungen (7,8\%). Als unabhängige Risikofaktoren für einen frühen Tod erwiesen sich BMI, diastolischer Blutdruck und Muskelkraft.

Männer, die bei den Krafttests punkteten, hatten sowohl eine niedrigere kardiovaskuläre Sterblichkeit als auch ein geringeres Gesamtmortalitätsrisiko. Das kardiovaskuläre Sterberisiko der Stärksten lag um 35\% unter dem der Teilnehmer der Referenzgruppe. Die Suizidraten waren um 20-30\% niedriger, und auch das Risiko für eine psychische Erkrankung lag um 15-65\% unter dem der Vergleichsgruppe.

Als zuverlässigstes Instrument für die Beurteilung der Muskelkraft hat sich der Handgrip-Strength-Test erwiesen. Die Autoren halten ihn in der Prävention für hilfreich, um schwachen Jugendlichen frühzeitig zu einer Verbesserung ihrer Fitness zu raten. ST .

Ortega FB et al. BMJ 2012;345:e7279; doi: 10.1136/ bmj.e7279

\section{ARZTBEWERTUNG IM INTERNET „Den meisten geht es nicht ums Meckern"}

- Patienten, die Arztbewertungsseiten im Internet nutzen, tun dies meist nicht, um gegen den Doktor zu wettern. In einer englischen Studie waren vor allem diejenigen bereit, einen Kommentar zu posten, die das Verhältnis zu ihrem Arzt als gut empfanden. Auf der Website "NHS Choices" der britischen Gesundheitsbehörde (National Health Service) können Patienten Lob oder Kritik im Zusammenhang mit einer ärztlichen Behandlung loswerden. Wer dieses Angebot nutzt und warum, wollten Forscher um M. Galizzi von der London School of Economics herausfinden. Dazu hatten sie 200 Londoner Bürger befragt.

Willig, einen Kommentar abzugeben oder das Informationsangebot der Seite zu nutzen, waren überraschenderweise gerade diejenigen, die Positives berichten konnten:
Ihr Arzt habe sich für sie Zeit genommen, er könne ihnen gut zuhören. Die Online-Kommentare drücken zudem sowohl Autonomie als auch medizinisches Interesse aus, fanden die Autoren.

Dagegen gebe es keinerlei Hinweis dafür, dass Bewertungen im Netz das Verhältnis zwischen Arzt und Patienten trüben könnten. „In unserer Studie war die Absicht, NHS Choices zu nutzen, keineswegs das Ergebnis einer unbefriedigenden Konsultation", schreiben Galizzi und Kollegen. Es gehe nicht darum, zu meckern oder Dampf abzulassen. Das Internet werde vielmehr als ergänzende Informationsquelle gesehen.

EO -

Galizzi MM et al. BMJ Open 2012;2:e001493; doi:10.1136/bmjopen-2012-001493 